\title{
Interferons, immunity and cancer immunoediting leading to impaired immune function in cancer patients
}

We read with great interest the recent article by Dunn et al. on the role of interferons (IFNs) in cancer immunosurveillance and sculpting of developing tumours, which they addressed as cancer immunoediting ${ }^{1}$. The contribution of IFNs to the dynamic relationship between the host immune system and cancer, and their central role as coordinators of tumour-immune-system interactions is reviewed in an excellent and comprehensive way. It provides compelling evidence that IFNs indeed protect the host against tumour development. However, the authors also discuss accumulating data that show that the immune system can promote tumour growth, with an obligatory role for IFNs in this process.

We decided to comment on this article because the role of IFNs in cancer immunosurveillance and tumour sculpting is an almost neglected issue in clinical practice. Until recently, IFNs had a solely positive image as probably the most important anti-proliferative cytokine in terms of tumour elimination. However, evidence indicates that IFNs are also involved in the ability of some tumours to escape immune detection, induce tolerance and impair immune functioning by activating a negative-feedback loop if the immune system is unable to eliminate the malignant tumour cells and if immune activation persists ${ }^{2}$.

Because of the outstanding potency of IFN $\gamma$, it is not surprising to find side effects both on administration and during clinical conditions of continuous immune stimulation, such as in cancer, in which endogenous IFN $\gamma$ production might exert a strong negative effect on host cells. Several antiproliferative biochemical pathways are induced by IFN $\gamma$; among them indoleamine 2,3-dioxygenase (IDO), a tryptophan-degrading enzyme, has been shown to affect T-cell responses ${ }^{3}$, and therefore can contribute to the development of immunodeficiency in patients with malignant disease. Furthermore, accelerated tryptophan degradation has been documented in various other clinical conditions, including infections, organ transplantation, autoimmune syndromes and in patients with coronary artery disease ${ }^{4}$. IDO activity is estimated by calculating the ratio of the IDO product kynurenine to its substrate tryptophan (the kynurenine-to-tryptophan ratio) and it is strongly associated with increased levels of the pteridine derivative neopterin in the blood of patients. Neopterin is released in large amounts by monocyte-derived macrophages after IFN $\gamma$ stimulation ${ }^{5}$. In cancer patients, accelerated tryptophan degradation and increased neopterin concentrations not only reflect disease progression, but were found to be associated with reduced survival and to correlate with levels of IFN $\gamma$ in the blood ${ }^{6}$. Therefore, in the clinical setting, the immunosuppressive role of IFNs and biochemical pathways that are triggered by IFNs become increasingly evident in chronic-disease states ${ }^{7}$.

Once this additional facet of IFN function is generally accepted, its role in the development of the symptoms that comprise the cancer cachexia syndrome, such as anaemia, metabolic changes, fatigue and neuropsychiatric disturbances, is obvious ${ }^{4}$. In addition, we recently showed that IDO expression indeed contributes to the cancer immunoediting process in humans ${ }^{7}$. In patients with colon carcinoma, enhanced IDO expression and increased tryptophan degradation resulted in a limitation of the antitumoural immune response and correlated with the frequency of metastases and overall survival of the patient, as well as with the number of tumour-infiltrating $\mathrm{T}$ cells ${ }^{7}$.

In conclusion, the diverse function of IFNs in cancer immunity fills an important gap in understanding the pathogenesis of long-term side effects of this important cytokine, leading to impaired immune function and immunodeficiency in cancer patients.

\section{Gerald Brandacher*, Raimund Margreiter* and Dietmar Fuchs ${ }^{\ddagger}$ \\ *Department of General and Transplant Surgery, Tyrolean Cancer Research Institute, Innsbruck Medical University, Anichstrasse 35, A-6020 Innsbruck, Austria.}

${ }^{ \pm}$Division of Biological Chemistry, Biocenter, Innsbruck Medical University, Fritz Pregl Strasse 3, A-6020 Innsbruck, Austria.

Correspondence to G.B. e-mail: gerald.brandacher@uki.at 
1. Dunn, G. P., Koebel C. M. \& Schreiber, R. D. Interferons, immunity and cancer immunoediting. Nature Rev. Immunol. 6, 836-848 (2006).

2. Brandacher, G., Winkler, C., Schroecksnadel, K., Margreiter, R. \& Fuchs, D. Antitumoral activity of interferon- $\gamma$ involved in impaired immune function in cancer patients. Curr. Drug Metab. 7, 599-612 (2006).

3. Mellor, A. L. \& Munn, D. H. IDO expression by dendritic cells: tolerance and tryptophan catabolism. Nature Rev. Immunol. 4, 762-774 (2004).

4. Wirleitner, B., Neurauter, G., Schroecksnadel, K., Frick, B. \& Fuchs, D. Interferon- $\gamma$-induced conversion of tryptophan: immunologic and neuropsychiatric aspects. Curr. Med. Chem. 10, $1581-1591(2003)$.

5. Fuchs, D. et al. Neopterin as a marker for activated cell-mediated immunity: application in HIV infection. Immunol. Today 9, 150-155 (1988).

6. Denz, H. et al. Correlation between neopterin, interferon- $\gamma$ and haemoglobin in patients with haematological disorders. Eur. J. Haematol. 44, 186-189 (1990).

7. Brandacher, G. et al. Prognostic value of indoleamine 2, 3-dioxygenase expression in colorectal cancer: effect on tumor-infiltrating T cells. Clin. Cancer Res. 12, 1144-1151 (2006). 